Pacific

Journal of

Mathematics

ON SOME POINTWISE INEQUALITIES CONCERNING TENT SPACES AND SHARP MAXIMAL FUNCTIONS

ANDREI K. LERNER 


\title{
ON SOME POINTWISE INEQUALITIES CONCERNING TENT SPACES AND SHARP MAXIMAL FUNCTIONS
}

\author{
ANDREI K. LERNER
}

We consider an abstract analogue of $S_{\lambda}^{\#} f$, the truncated square function introduced by J.-O. Strömberg, and show that it is closely related to operators appearing in the theory of tent spaces. We suggest an approach to basic results for these spaces which differs from that due to R.R. Coifman, Y. Meyer and E.M. Stein. Also we discuss pointwise estimates involving $S_{\lambda}^{\#} f$ as well as different variants of sharp maximal functions.

\section{Introduction.}

Let $\mathbb{R}_{+}^{n+1}=\left\{(y, t): y \in \mathbb{R}^{n}, t>0\right\}$ be the upper half space. For $\alpha, h>0$ define the truncated cone $\Gamma_{\alpha}^{h}(x)=\left\{(y, t) \in \mathbb{R}_{+}^{n+1}:|y-x|<\alpha t, 0<t<h\right\}$. Let $\Gamma_{\alpha}(x)=\Gamma_{\alpha}^{\infty}(x)$. When $\alpha=1$ we simply write $\Gamma^{h}(x), \Gamma(x)$. Given a ball $B=B(x, r)$ in $\mathbb{R}^{n}$ centered at $x$ of radius $r$, denote by $T(B)$ the tent over $B$, that is, $T(B)=\{(y, t):|y-x|+t \leq r\}$. For two quantities $a, b$, we write $a \asymp b$ if there exist absolute constants $c_{1}, c_{2}$ such that $c_{1} a \leq b \leq c_{2} a$.

For any measurable function $F$ defined on $\mathbb{R}_{+}^{n+1}$, set

$$
A(F \mid h)(x)=\int_{\Gamma^{h}(x)}|F(y, t)| \frac{d y d t}{t^{n+1}},
$$

and

$$
\mathcal{C} F(x)=\sup _{B \ni x} \frac{1}{|B|} \int_{T(B)}|F(y, t)| \frac{d y d t}{t},
$$

where the sup is taken over all balls $B$ containing $x$. Let $A F=A(F \mid \infty)$; let for $q>0, A_{q} F(x)=\left(A|F|^{q}(x)\right)^{1 / q}$ and $\mathcal{C}_{q} F(x)=\left(\mathcal{C}|F|^{q}(x)\right)^{1 / q}$. Set

$$
A_{\infty} F(x)=\sup _{(y, t) \in \Gamma(x)}|F(y, t)| .
$$

In [4], R.R. Coifman, Y. Meyer, and E.M. Stein introduced the tent spaces $T_{q}^{p}=T_{q}^{p}\left(\mathbb{R}_{+}^{n+1}\right)$. These spaces provide a very useful tool for solving problems in harmonic analysis. When $0<p, q<\infty$ the space $T_{q}^{p}$ consists of all $F$ such that

$$
\|F\|_{T_{q}^{p}}=\left\|A_{q} F\right\|_{L^{p}\left(\mathbb{R}^{n}\right)}<\infty
$$


The space $T_{\infty}^{p}(p<\infty)$ is defined as the space of continuous functions $F$ which have non-tangential boundary limits a.e., and such that

$$
\|F\|_{T_{\infty}^{p}}=\left\|A_{\infty} F\right\|_{L^{p}\left(\mathbb{R}^{n}\right)}<\infty .
$$

The space $T_{q}^{\infty}(q<\infty)$ is defined by requiring that

$$
\|F\|_{T_{q}^{\infty}}=\left\|\mathcal{C}_{q} F\right\|_{L^{\infty}\left(\mathbb{R}^{n}\right)}<\infty .
$$

Under the latter definition, the pairing $\langle F, G\rangle=\int_{\mathbb{R}_{+}^{n+1}} F(y, t) G(y, t) d y d t / t$ realizes $T_{q^{\prime}}^{\infty}$ as equivalent to the dual of $T_{q}^{1}$. Also the above pairing gives that the dual of $T_{q}^{p}$ is $T_{q^{\prime}}^{p^{\prime}}$, where $1 \leq p<\infty, 1<q<\infty$, and, as usual, $1 / p+1 / p^{\prime}=1$ and $1 / q+1 / q^{\prime}=1$.

In this paper we show that, besides $A F$ and $\mathcal{C} F$, in the study of the tent spaces the operator $A_{\lambda}^{\#} F$, defined by

$$
A_{\lambda}^{\#} F(x)=\sup _{B \ni x}\left(\left(A\left(F \mid r_{B}\right)\right) \chi_{B}\right)^{*}(\lambda|B|) \quad(0<\lambda<1),
$$

is also of considerable interest. Here the sup is taken over all balls $B$ containing $x, r_{B}$ and $\chi_{B}$ denote the radius and the indicator function of $B$, respectively, and $f^{*}(t)$ denotes the standard non-increasing rearrangement of $f$. For $q>0$ set $A_{q, \lambda}^{\#} F(x)=\left(A_{\lambda}^{\#}|F|^{q}(x)\right)^{1 / q}$. Note that the function $A_{q, \lambda}^{\#} F$ is particularly interesting if $q=2$ and $F$ is the Poisson integral of $f, F=f * P_{t}(y)$. We denote such a function by $S_{\lambda}^{\#} f$ and consider it below in connection with some pointwise estimates for $A_{q, \lambda}^{\#} F$. (Note that the function $S_{\lambda}^{\#} f$ was introduced and considered by J.-O. Strömberg in [14].)

The paper is organized as follows: In Section 2 we state and discuss our main results which we prove in Section 4 . Section 3 contains some additional notation and auxilary results.

\section{Main results: Formulations and discussion.}

Let us discuss our main results and their applications to the theory of the tent spaces.

2.1. Relations between the operators $A_{q} F, \mathcal{C}_{q} F$ and $A_{q, \lambda}^{\#} F$. It is shown in [4] that the operator $\mathcal{C} F$ can be used to give an alternate definition of $T_{q}^{p}$ when $0<q<p<\infty$, since in this case

$$
\left\|A_{q} F\right\|_{p} \asymp\left\|\mathcal{C}_{q} F\right\|_{p} .
$$

Roughly speaking, our first theorem investigates the relations between $A_{q} F, \mathcal{C}_{q} F$ and $A_{q, \lambda}^{\#} F$, and, in particular, allows us to define the spaces $T_{q}^{p}$ in terms of $A_{\lambda}^{\#} F$ for all $0<q<\infty, 0<p \leq \infty$. 
Theorem 2.1. Let $F$ be any measurable function defined on $\mathbb{R}_{+}^{n+1}$.

a) For all $0<q<\infty$ and $x \in \mathbb{R}^{n}$,

$$
\mathcal{C}_{q} F(x) \asymp M_{q} A_{\lambda, q}^{\#} F(x) \quad(0<\lambda<1),
$$

where $M_{q} f=\left(M|f|^{q}\right)^{1 / q}$ and $M$ is the Hardy-Littlewood maximal function.

b) For all $1 \leq q<\infty$ and $t>0$,

$$
\left(A_{q} F\right)^{*}(t) \leq c_{1} \int_{c_{2} t}^{\infty}\left(A_{q, \lambda}^{\#} F\right)^{*}(\tau) \frac{d \tau}{\tau} \quad(0<\lambda<1),
$$

where $c_{1}, c_{2}$ depend only on $\lambda$ and $n$.

Some comments about these results are in order. For any measurable function $f$ on $\mathbb{R}^{n}$ consider the maximal function $m_{\lambda} f$ defined by

$$
m_{\lambda} f(x)=\sup _{B \ni x}\left(f \chi_{B}\right)^{*}(\lambda|B|) \quad(0<\lambda<1) .
$$

Since $\left\{m_{\lambda} f>\alpha\right\} \subset\left\{M \chi_{\{|f|>\alpha\}} \geq \lambda\right\}$, we have $\left(m_{\lambda} f\right)^{*}(t) \leq f^{*}\left(c_{\lambda, n} t\right)$, and hence, for all $p>0$,

$$
\left\|m_{\lambda} f\right\|_{p} \leq c_{p, \lambda, n}\|f\|_{p} .
$$

Clearly, $A_{q, \lambda}^{\#} F(x) \leq m_{\lambda}\left(A_{q} F\right)(x)$, and so, $\left\|A_{q, \lambda}^{\#} F\right\|_{p} \leq c_{p, \lambda, n}\left\|A_{q} F\right\|_{p}$ for all $p, q>0$. Using the duality argument, one can show that the converse also holds. However, the combining of (3) and classical Hardy's inequality (see, e.g., [2, p. 124]) immediately gives a direct proof of this fact. Moreover, taking into account (2), we obtain that one can characterize the spaces $T_{q}^{p}$ in terms of $A_{\lambda}^{\#} F$ :

$$
\|F\|_{T_{q}^{p}} \asymp\left\|A_{\lambda, q}^{\#} F\right\|_{p} \quad(0<q<\infty, 0<p \leq \infty, 0<\lambda<1) .
$$

Inequality (1) is the other corollary of this theorem, which follows from (2), (3) and the Hardy-Littlewood maximal theorem.

Define the maximal function $M_{\lambda}^{\#} f$ by

$$
M_{\lambda}^{\#} f(x)=\sup _{Q \ni x} \inf _{c \in \mathbb{R}}\left((f-c) \chi_{Q}\right)^{*}(\lambda|Q|) \quad(0<\lambda<1),
$$

where the sup is taken over all cubes $Q$ containing $x$. In [11], the following rearrangement inequality was obtained for all $t>0$ and any measurable function $f$ with $f^{*}(+\infty)=0$ :

$$
f^{*}(t) \leq \frac{2}{\log 2} \int_{t}^{\infty}\left(M_{\lambda}^{\#} f\right)^{*}(\tau) \frac{d \tau}{\tau} \quad\left(0<\lambda<\lambda_{n}\right) .
$$

This will be a key tool in proving (3).

It is worth noting that the function $M_{\lambda}^{\#} f$ was also introduced in the above mentioned paper [14], where its definition was motivated by an alternate 
characterization of the space $B M O$. Recall that $B M O$ consists of all locally integrable functions $f$ on $\mathbb{R}^{n}$ such that

$$
\|f\|_{*}=\sup _{Q} \frac{1}{|Q|} \int_{Q}\left|f(x)-f_{Q}\right| d x<\infty \quad\left(f_{Q}=\frac{1}{|Q|} \int_{Q} f\right) .
$$

By Chebyshev's inequality, it is clear that $\lambda\left\|M_{\lambda}^{\#} f\right\|_{\infty} \leq\|f\|_{*}$. However, F. John [10] for $0<\lambda<1 / 2$ and J.-O. Strömberg [14] for $\lambda=1 / 2$ proved that the converse is also true:

$$
\|f\|_{*} \leq c_{n}\left\|M_{\lambda}^{\#} f\right\|_{\infty} \quad(0<\lambda \leq 1 / 2) .
$$

2.2. Real interpolation of tent spaces. It is proved in [4] that

$$
\left(T_{q}^{p_{0}}, T_{q}^{p_{1}}\right)_{\theta, p}=T_{q}^{p} \quad(1<q<\infty),
$$

where $0<\theta<1,1 \leq p_{0}<p_{1} \leq \infty$ and $1 / p=(1-\theta) / p_{0}+\theta / p_{1}$, and $(\cdot, \cdot)_{\theta, p}$ is the real method of interpolation (cf. [2, p. 299]). A different proof of (7) is given by J. Alvarez and M. Milman [1]. Generally speaking, both proofs consist of two main steps: First (7) is proved under certain constraints on the interval for $p_{0}, p_{1}$ (without end-points), then the result is extended to the whole range of $p_{0}, p_{1}$ by the duality and Wolff's reiteration theorem.

Here we use our approach to prove (7) via sharp estimates for the $K$ functional. Namely, in our next result we state that in the end-point case Peetre's $K$-functional for the couple $\left(T_{q}^{1}, T_{q}^{\infty}\right)$ is explicitly characterized in terms of $A_{q, \lambda}^{\#} F$. Our proof works for all $0<q<\infty$, so (7) can be extended to the case $0<q \leq 1$. Recall that the $K$-functional is defined as

$$
K\left(F, t ; T_{q}^{1}, T_{q}^{\infty}\right)=\inf _{F=F_{1}+F_{2}}\left(\left\|F_{1}\right\|_{T_{q}^{1}}+t\left\|F_{2}\right\|_{T_{q}^{\infty}}\right)
$$

for all $F \in T_{q}^{1}+T_{q}^{\infty}$ and $t>0$.

Theorem 2.2. Let $0<q<\infty$. Then for all $F \in T_{q}^{1}+T_{q}^{\infty}$ and $t>0$,

$$
K\left(F, t ; T_{q}^{1}, T_{q}^{\infty}\right) \asymp \int_{0}^{t}\left(A_{q, \lambda}^{\#} F\right)^{*}(\tau) d \tau \quad(0<\lambda<1) .
$$

This theorem along with (5) easily implies (7) in the case $p_{0}=1, p_{1}=\infty$ for all $0<q<\infty$. Now one can apply the Holmstedt reiteration theorem (see, e.g., [2, p. 307]) to describe the K-functional for any of the couples $\left(T_{q}^{p_{0}}, T_{q}^{p_{1}}\right)$ and get $(7)$ for all $1<p_{0}<p_{1}<\infty$.

2.3. Factorization of tent spaces. For the tent spaces the following factorization holds:

$$
T_{q}^{p}=T_{\infty}^{p} \cdot T_{q}^{\infty}
$$


This fact was proved in [4] in the case $p>2$ and $q=2$. Recently, W.S. Cohn and I.E. Verbitsky [3] have extended (8) to all $0<p, q<\infty$. This result is partially based on the next inequality $[\mathbf{3}]$ :

$$
\|F G\|_{T_{q}^{p}} \leq c_{p, q}\|F\|_{T_{\infty}^{p}}\|G\|_{T_{q}^{\infty}} .
$$

We propose a different proof of (9). It follows immediately from (4), (5) and the next elementary estimate.

Proposition 2.3. For any functions $F, G$ defined on $\mathbb{R}_{+}^{n+1}$ and for all $x$,

$$
A_{q, \lambda}^{\#}(F G)(x) \leq m_{\lambda / 4}\left(A_{\infty} F\right)(x) A_{q, \lambda / 4}^{\#} G(x) \quad(0<\lambda<1) .
$$

2.4. Pointwise estimates for $S_{\lambda}^{\#} f$. Let us return to the function $S_{\lambda}^{\#} f$ and discuss several pointwise estimates motivated by (2) and the well-known C. Fefferman's duality theorem. We consider the definition of $S_{\lambda}^{\#} f$ in the following slightly generalized form. Let $\varphi$ be a real-valued differentiable function on $\mathbb{R}^{n}$ which satisfies:

$$
\begin{aligned}
& |\varphi(x)| \leq c(1+|x|)^{-n-1},|\nabla \varphi(x)| \leq c(1+|x|)^{-n-1}, \\
& \int_{\mathbb{R}^{n}} \varphi(x) d x=0 .
\end{aligned}
$$

Write $\varphi_{t}(x)=\varphi(x / t) t^{-n}, t>0$. Given a function $f$ with

$$
\int_{\mathbb{R}^{n}}|f(x)|(1+|x|)^{-n-1} d x<\infty,
$$

set $F(y, t)=\left|f * \varphi_{t}(y)\right|$, and define $S_{\lambda}^{\#} f$ by (cf. [14])

$$
S_{\lambda}^{\#} f(x)=A_{2, \lambda}^{\#} F(x) \text {. }
$$

Denote by $\mathcal{S}\left(\mathbb{R}^{n}\right)$ the class of Schwartz functions on $\mathbb{R}^{n}$. Assume that, in addition to (i) and (ii), $\varphi$ satisfies

(iii) there exists a function $\psi \in \mathcal{S}\left(\mathbb{R}^{n}\right)$ such that

$$
\begin{gathered}
\int_{\mathbb{R}^{n}} \psi(x) d x=0, \operatorname{supp} \psi \subset\{|x| \leq 1\} \quad \text { and } \\
\int_{0}^{\infty} \widehat{\varphi}(t \xi) \widehat{\psi}(t \xi) \frac{d t}{t} \equiv 1 \quad \text { for all } \xi \neq 0 .
\end{gathered}
$$

In particular, $\varphi$ satisfies (iii) whenever $\varphi$ is radial and $\widehat{\varphi}(\xi) \geq 0$ [13, p. 186]. Define the maximal function $\mathcal{F}^{\#} f$ by

$$
\mathcal{F}^{\#} f(x)=\mathcal{C}_{2} F(x),
$$

where, as above, $F(y, t)=\left|f * \varphi_{t}(y)\right|$. If $\varphi$ satisfies (i)-(iii), then one of the equivalent formulations of $\mathrm{C}$. Fefferman's duality theorem (see [5] and [6] or $\left[13\right.$, p. 159]) states that $f \in B M O \Leftrightarrow \mathcal{F}^{\#} f \in L^{\infty}$ and

$$
\|f\|_{*} \asymp\left\|\mathcal{F}^{\#} f\right\|_{\infty} .
$$


By (2), we see that $\mathcal{F}^{\#} f(x) \asymp M_{2} S_{\lambda}^{\#} f(x)$, and therefore $\|f\|_{*} \asymp\left\|S_{\lambda}^{\#} f\right\|_{\infty}$. In view of (3), we also obtain that for all $p>0$,

$$
\|S f\|_{p} \asymp\left\|S_{\lambda}^{\#} f\right\|_{p} \quad(0<\lambda<1),
$$

where $S f$ is the Lusin area integral. Hence,

$$
\|f\|_{H^{p}} \asymp\left\|S_{\lambda}^{\#} f\right\|_{L^{p}} \quad(0<\lambda<1) .
$$

This was proved by B. Jawerth [8]. His proof was based on atomic decomposition. Note that the following characterization:

$$
K\left(f, t ; H^{1}, B M O\right) \asymp \int_{0}^{t}\left(S_{\lambda}^{\#} f\right)^{*}(\tau) d \tau
$$

was also established in [8]. It is interesting to compare this result with Theorem 2.2.

Observe that inequality (2) may be viewed as an analogue of the following two-sided estimate proved by B. Jawerth and A. Torchinsky [9]:

$$
(f)_{q}^{\#}(x) \asymp M_{q} M_{\lambda}^{\#} f(x) \quad\left(0<\lambda<\lambda_{n}\right),
$$

whenever $q>0$ and $f \in L^{q}+B M O$, where

$$
(f)_{q}^{\#}(x)=\sup _{Q \ni x}\left(\frac{1}{|Q|} \int_{Q}\left|f(y)-f_{Q}\right|^{q} d y\right)^{1 / q} .
$$

A natural question arises from this: What is a pointwise connection between functions $M_{\lambda}^{\#} f$ and $S_{\lambda}^{\#} f$ ? The following estimate answers the question in one direction:

$$
S_{\lambda}^{\#} f(x) \leq c f^{\#}(x) .
$$

In essense, it was proved in [14]. It is clear that the reverse inequality fails (e.g., for $f \in H^{p}, p \leq 1$ ). Nevertheless, using the quasi-orthogonal decomposition of $f[\mathbf{1 3}$, p. 166], we prove:

Theorem 2.4. Suppose $f$ satisfies (10) and $\varphi$ satisfies (i)-(iii). Then for all $x \in \mathbb{R}^{n}$,

$$
M_{\lambda}^{\#} f(x) \leq c S_{\lambda}^{\#} f(x) \quad\left(0<\lambda<\lambda_{n}\right),
$$

where $c$ depends on $\lambda$ and $n$.

This inequality also can not be reversed (e.g., for $f \in L^{p} \backslash H^{p}, p \leq 1$ ). However, using inequalities (2), (12-14), we obtain the following "pointwise" version of C. Fefferman's theorem:

Corollary 2.5. Let $f$ satisfies (10) and $\varphi$ satisfies (i)-(iii). Then

$$
\mathcal{F}^{\#} f(x) \asymp M_{2} S_{\lambda}^{\#} f(x) \asymp M_{2} M_{\lambda}^{\#} f(x) \asymp(f)_{2}^{\#}(x) \quad\left(0<\lambda<\lambda_{n}\right) .
$$




\section{Preliminaries.}

We say that $f^{*}$ is the non-increasing rearrangement of $f$ if it is non-increasing on $(0,+\infty)$ and equimeasurable with $|f(x)|$. We shall assume that the rearrangement is left-continuous. Then it is uniquely determined and can be defined by the equality

$$
f^{*}(t)=\sup _{|E|=t} \inf _{x \in E}|f(x)|
$$

Throughout the paper, we shall use the following simple inequality (see, e.g., $[2$, p. 41]):

$$
(f+g)^{*}\left(t_{1}+t_{2}\right) \leq f^{*}\left(t_{1}\right)+g^{*}\left(t_{2}\right) .
$$

We will prove one more property of rearrangements, though it is apparently known. For any measurable set $E \subset \mathbb{R}^{n}$ we shall denote its complement $\mathbb{R}^{n} \backslash E$ by $E^{c}$.

Proposition 3.1. Let $\alpha+\beta<t$. Then for any measurable functions $f, g$,

$$
(f g)^{*}(t) \leq f^{*}(\alpha) g^{*}(\beta) \text {. }
$$

Proof. Let $E_{1}=\left\{x:|f(x)| \leq f^{*}(\alpha)\right\}, E_{2}=\left\{x:|g(x)| \leq g^{*}(\beta)\right\}$. Then $\left|E_{1}^{c} \cup E_{2}^{c}\right| \leq \alpha+\beta$. Thus, for any measurable set $E \subset \mathbb{R}^{n}$ with $|E|=t$ we have $\left|E \cap\left(E_{1} \cap E_{2}\right)\right|>0$, and so

$$
\inf _{x \in E}|f g(x)| \leq f^{*}(\alpha) g^{*}(\beta),
$$

which completes the proof.

Now, let us define

$$
A^{\prime}(F \mid h)(x)=\int_{\Gamma_{2}^{h}(x)}|F(y, t)| \frac{d y d t}{t^{n+1}} .
$$

We shall need two following lemmas:

Lemma 3.2. Let $\mathfrak{F} \subset \mathbb{R}^{n}$ be an arbitrary closed set whose complement $\mathfrak{F}^{c}$ has finite measure. There is a subset $\mathfrak{F}^{*} \subset \mathfrak{F}$ such that $\left|\mathfrak{F}^{* c}\right| \leq c_{n}\left|\mathfrak{F}^{c}\right|$, and for any non-negative $F$ the following inequality holds:

$$
\int_{\cup_{x \in \mathfrak{F}^{*}}} F(y, t) t^{n} d y d t \leq c_{n}^{\prime} \int_{\mathfrak{F}}\left(\int_{\Gamma(x)} F(y, t) d y d t\right) d x .
$$

This result is well-known (see [4] or [13, p. 126]).

Lemma 3.3. For any ball $B$ containing $x$, we have

$$
\left(\left(A^{\prime}\left(F \mid r_{B}\right)\right) \chi_{B}\right)^{*}(\lambda|B|) \leq c_{1} A_{c_{2} \lambda}^{\#} F(x) \quad(0<\lambda<1),
$$

where $c_{1}$ depends on $\lambda$ and $n$, while $c_{2}$ depends only on $n$. 
Proof. Set $D=\bigcup_{x \in B} \Gamma_{2}^{r_{B}}(x)$ and

$$
\mathfrak{F}=\left\{x: A\left(F \chi_{D}\right)(x) \leq\left(A\left(F \chi_{D}\right)\right)^{*}\left(\lambda|B| / 2 c_{n}\right)\right\},
$$

where $c_{n}$ is the same as in Lemma 3.2. A simple geometric argument shows that $A\left(F \chi_{D}\right)$ is supported in $8 B$. Thus $\left(A\left(F \chi_{D}\right)\right)^{*}\left(\lambda|B| / 2 c_{n}\right) \leq A_{c \lambda}^{\#} F(x)$. Let $E \subset B$ be an arbitrary measurable set with $|E|=\lambda|B|$. Choose $\mathfrak{F}^{*} \subset \mathfrak{F}$ as in Lemma 3.2. Then $\left|\mathfrak{F}^{* c}\right| \leq c_{n}\left|\mathfrak{F}^{c}\right| \leq \lambda|B| / 2$, and hence $\left|\mathfrak{F}^{*} \cap E\right| \geq \lambda|B| / 2$. Applying Lemma 3.2 and Fubini's theorem, we get

$$
\begin{aligned}
\inf _{\xi \in E} A^{\prime}\left(F \mid r_{B}\right)(\xi) & \leq \inf _{\xi \in \mathfrak{F}^{*} \cap E} A^{\prime}\left(F \chi_{D}\right)(\xi) \leq \frac{2}{\lambda|B|} \int_{\mathfrak{F}^{*} \cap E} A^{\prime}\left(F \chi_{D}\right)(\xi) d \xi \\
& \leq \frac{c}{|B|} \int_{\bigcup_{\xi \in \mathfrak{F}^{*} \Gamma_{2}(\xi)}}\left|F \chi_{D}(y, t)\right| \frac{d y d t}{t} \\
& \leq \frac{c}{|B|} \int_{\mathfrak{F}} A\left(F \chi_{D}\right)(\xi) d \xi \leq c_{1} A_{c_{2} \lambda}^{\#} F(x) .
\end{aligned}
$$

To complete the proof take the sup over all $E \subset B$ with $|E|=\lambda|B|$.

Also in this section we recall some useful ideas when dealing with $S_{\lambda}^{\#} f$. Suppose $f$ satisfies (10) and $\varphi$ satisfies (i)-(ii). Set

$$
S_{h} f(x)=\left(\int_{\Gamma^{h}(x)}\left|f * \varphi_{t}(y)\right|^{2} \frac{d y d t}{t^{n+1}}\right)^{1 / 2}, \quad S f(x)=S_{\infty} f(x) .
$$

Let $x \in B$ and let $f_{1}=f \chi_{4 B}, f_{2}=f \chi_{(4 B)^{c}}$. Since $S$ is of weak type $(1,1)$ (see $[\mathbf{1 2}]$ ), we have

$$
\left(\left(S_{r_{B}} f_{1}\right) \chi_{B}\right)^{*}(\lambda|B|) \leq\left(S f_{1}\right)^{*}(\lambda|B|) \leq \frac{c}{\lambda|B|} \int_{4 B}|f| .
$$

Further, standard arguments (see, e.g., [13, p. 160]) yield

$$
\left|f_{2} * \varphi_{t}(y)\right| \leq c \int_{\mathbb{R}^{n} \backslash 4 B}|f(\xi)| \frac{t}{\left(r_{B}+|x-\xi|\right)^{n+1}} d \xi,
$$

whenever $x \in B$ and $(y, t) \in \cup_{\eta \in B} \Gamma_{r_{B}}(\eta)$, and hence,

$$
\begin{aligned}
& \left(\left(S_{r_{B}} f_{2}\right) \chi_{B}\right)^{*}(\lambda|B|) \\
& \leq c\left(\frac{1}{|B|} \int_{T(3 B)}\left(t / r_{B}\right)^{2} \frac{d y d t}{t}\right)^{1 / 2} \int_{\mathbb{R}^{n} \backslash 4 B}|f(\xi)| \frac{r_{B}}{\left(r_{B}+|x-\xi|\right)^{n+1}} d \xi \\
& \leq c \int_{\mathbb{R}^{n} \backslash 4 B}|f(\xi)| \frac{r_{B}}{\left(r_{B}+|x-\xi|\right)^{n+1}} d \xi .
\end{aligned}
$$

To extend (14) from Schwartzian functions to those satisfying (10) in the proof of Theorem 2.4, we use that $\int_{\mathbb{R}^{n}}\left|f_{j}(\xi)\right|(1+|\xi|)^{-n-1} d \xi \rightarrow 0$ implies $\left(S_{r_{B}} f_{j}\right)^{*}(\lambda|B|) \rightarrow 0$ as $j \rightarrow \infty$. This readily follows from (15) and (16). 
Observe also that taking $f_{1}=\left(f-f_{4 B}\right) \chi_{4 B}, f_{2}=\left(f-f_{4 B}\right) \chi_{(4 B)^{c}}$ yields that the right sides of (15) and (16) are at most $c_{\lambda, n} f^{\#}(x)$. Since $\int \varphi=0$, we have $S_{r_{B}} f \leq \sqrt{2}\left(S_{r_{B}} f_{1}+S_{r_{B}} f_{2}\right)$, and, by (15) and (16),

$$
\begin{aligned}
\left(\left(S_{r_{B}} f\right) \chi_{B}\right)^{*}(\lambda|B|) \leq & \sqrt{2}\left(\left(S_{r_{B}} f_{1}\right) \chi_{B}\right)^{*}(\lambda|B| / 2) \\
& +\sqrt{2}\left(\left(S_{r_{B}} f_{2}\right) \chi_{B}\right)^{*}(\lambda|B| / 2) \leq c_{\lambda, n} f^{\#}(x),
\end{aligned}
$$

which gives (13).

\section{Proofs of the main results.}

First of all, let us show that, as we mentioned after (4), the inequality

$$
\left\|A_{q} F\right\|_{p} \leq c_{\lambda, p, q}\left\|A_{q, \lambda}^{\#} F\right\|_{p} \quad(0<p, q<\infty, 0<\lambda<1)
$$

can be derived by duality. Suppose first that $1 \leq p<\infty, 1<q<\infty$. We use the following standard argument: For $q>0$ define the "stopping-time" $h(x)$ by

$$
h(x)=\sup \left\{h>0: A_{q}(F \mid h)(x) \leq A_{q, \lambda}^{\#} F(x)\right\} .
$$

Then $A_{q}(F \mid h(x))(x) \leq A_{q, \lambda}^{\#} F(x)$ and $\left|\left\{x \in B: h(x)>r_{B}\right\}\right| \geq(1-\lambda)|B|$ for each $B$. By Fubini's theorem and Hölder's inequality we have

$$
\begin{aligned}
\int_{\mathbb{R}_{+}^{n+1}}|F(y, t) G(y, t)| \frac{d y d t}{t} & \leq \frac{1}{1-\lambda} \int_{\mathbb{R}^{n}} \int_{\Gamma^{h(x)}(x)}|F(y, t) G(y, t)| \frac{d y d t}{t^{n+1}} d x \\
& \leq \frac{1}{1-\lambda} \int_{\mathbb{R}^{n}} A_{q}(F \mid h(x))(x) A_{q^{\prime}} G(x) d x \\
& \leq \frac{1}{1-\lambda} \int_{\mathbb{R}^{n}} A_{q, \lambda}^{\#} F(x) A_{q^{\prime}} G(x) d x
\end{aligned}
$$

(note that in [4] different variants of such inequality were obtained with $\mathcal{C}_{q} F$ or $A_{q} F$ instead of $A_{q, \lambda}^{\#} F$ on the right side). Applying Hölder's inequality again, we get

$$
\int_{\mathbb{R}_{+}^{n+1}}|F(y, t) G(y, t)| \frac{d y d t}{t} \leq \frac{1}{1-\lambda}\|G\|_{T_{q^{\prime}}^{p^{\prime}}}\left\|A_{q, \lambda}^{\#} F\right\|_{p},
$$

which gives (17) by duality. The restrictions on $p, q$ are easily removed by replacing $|F|$ by $|F|^{\delta}$ with appropriate $\delta>0$.

Proof of the first part of Theorem 2.1. It is clear that it suffices to consider the case $q=1$. Let $q=1$ and let $h$ as above. Then for $0<t<r_{B}, y \in B$ we get

$$
\left|\left\{x \in 3 B:(y, t) \in \Gamma^{h(x)}(x)\right\}\right| \geq c_{n}(1-\lambda) t^{n},
$$


where $c_{n}$ denotes the volume of the unit ball in $\mathbb{R}^{n}$. Applying Fubini's theorem gives

$$
\begin{aligned}
\int_{T(B)}|F(y, t)| \frac{d y d t}{t} & \leq \frac{1}{c_{n}(1-\lambda)} \int_{3 B} A(F \mid h(x))(x) d x \\
& \leq \frac{1}{c_{n}(1-\lambda)} \int_{3 B} A_{\lambda}^{\#} F(x) d x,
\end{aligned}
$$

and thus $\mathcal{C} F(x) \leq c_{\lambda, n} M A_{\lambda}^{\#} F(x)$.

Let us prove the converse. Let $x, \xi \in B$ and let $B^{\prime}$ be an arbitrary ball containing $\xi$. If $B \subset 3 B^{\prime}$, then

$$
\begin{aligned}
& \left(\left(A\left(F \mid r_{B^{\prime}}\right)\right) \chi_{B^{\prime}}\right)^{*}\left(\lambda\left|B^{\prime}\right|\right) \\
& \leq \frac{1}{\lambda\left|B^{\prime}\right|} \int_{B^{\prime}} A\left(F \mid r_{B^{\prime}}\right)(x) d x \\
& \leq \frac{c}{\left|B^{\prime}\right|} \bigcup_{x \in B^{\prime}} \int_{\Gamma^{r} B^{\prime}(x)}|F(y, t)| \frac{d y d t}{t} \leq c \mathcal{C} F(x),
\end{aligned}
$$

since $\bigcup_{x \in B^{\prime}} \Gamma^{r_{B^{\prime}}}(x) \subset T\left(3 B^{\prime}\right)$. Assume now that $B \not \subset 3 B^{\prime}$. Then $B^{\prime} \subset 3 B$ and in this case

$$
\left(\left(A\left(F \mid r_{B^{\prime}}\right)\right) \chi_{B^{\prime}}\right)^{*}\left(\lambda\left|B^{\prime}\right|\right) \leq m_{\lambda}\left(\left(A\left(F \mid 3 r_{B}\right)\right) \chi_{3 B}\right)(\xi) .
$$

Therefore, for all $\xi \in B$,

$$
A_{\lambda}^{\#} F(\xi) \leq c \mathcal{C} F(x)+m_{\lambda}\left(\left(A\left(F \mid 3 r_{B}\right)\right) \chi_{3 B}\right)(\xi) .
$$

Using (4), we get

$$
\begin{aligned}
\frac{1}{|B|} \int_{B} A_{\lambda}^{\#} F(\xi) d \xi & \leq c \mathcal{C} F(x)+\frac{1}{|B|}\left\|m_{\lambda}\left(\left(A\left(F \mid 3 r_{B}\right)\right) \chi_{3 B}\right)\right\|_{1} \\
& \leq c \mathcal{C} F(x)+\frac{c}{|B|} \int_{3 B} A\left(F \mid 3 r_{B}\right)(x) d x \leq c \mathcal{C} F(x) .
\end{aligned}
$$

Hence $M A_{\lambda}^{\#} F(x) \leq c_{\lambda, n} \mathcal{C} F(x)$, and (2) is proved.

Proof of the second part of Theorem 2.1. Choose a function $\Phi \in \mathcal{S}\left(\mathbb{R}^{n}\right)$ such that $\chi_{B}(0,1) \leq \Phi \leq \chi_{B}(0,3 / 2)$, and define

$$
\widetilde{A}(F)(x)=\int_{0}^{\infty} \int_{\mathbb{R}^{n}}|F(y, t)| \Phi_{t}(x-y) \frac{d y d t}{t} .
$$

Clearly, $A(F)(x) \leq \widetilde{A}(F)(x)$. The crucial observation to prove (3) is that

$$
M_{\lambda}^{\#}(\widetilde{A}(F))(x) \leq c A_{c^{\prime} \lambda}^{\#} F(x) \quad(0<\lambda<1) .
$$


Let $x \in Q, d_{Q}$ and $x_{Q}$ denote the diameter and center of $Q$ respectively, and

$$
c_{Q}=\int_{2 d_{Q}}^{\infty} \int_{\mathbb{R}^{n}}|F(y, t)| \Phi_{t}\left(x_{Q}-y\right) \frac{d y d t}{t} .
$$

Then, for $z \in Q$ we have

$$
\begin{aligned}
& \left|\int_{2 d_{Q}}^{\infty} \int_{\mathbb{R}^{n}}\right| F(y, t)\left|\Phi_{t}(z-y) \frac{d y d t}{t}-c_{Q}\right| \\
& \leq c d_{Q} \sum_{k=0}^{\infty} \int_{2^{k+1} d_{Q}} \int_{B(z, 3 t / 2) \cup B\left(x_{Q}, 3 t / 2\right)}|F(y, t)| \frac{d y d t}{t^{n+2}} .
\end{aligned}
$$

Note that for all $z \in Q, \xi \in 2^{k} Q$ and $t \geq 2^{k+1} d_{Q}$ we get $|z-\xi| \leq 2^{k} d_{Q} \leq t / 2$, and hence $B(z, 3 t / 2) \cup B\left(x_{Q}, 3 t / 2\right) \subset B(\xi, 2 t)$. It follows from this and Lemma 3.3 that the right side of (19) is at most

$$
\begin{aligned}
& c \sum_{k=0}^{\infty} \frac{1}{2^{k}} \inf _{\xi \in 2^{k} Q} \int_{\Gamma_{2}^{2^{k+2} d_{Q}(\xi)}}|F(y, t)| \frac{d y d t}{t^{n+1}} \\
& \leq c \sum_{k=0}^{\infty} \frac{1}{2^{k}}\left(\left(A^{\prime}\left(F \mid 2^{k+2} d_{Q}\right)\right) \chi_{2^{k} Q}\right)^{*}\left(2^{k}|Q|\right) \leq c A_{\lambda_{n}}^{\#} F(x) .
\end{aligned}
$$

Therefore, using Lemma 3.3 again, we get

$$
\begin{aligned}
& \left(\left(\widetilde{A}(F)-c_{Q}\right) \chi_{Q}\right)^{*}(\lambda|Q|) \\
& \leq\left(\left(A^{\prime}\left(F \mid 2 d_{Q}\right)\right) \chi_{Q}\right)^{*}(\lambda|Q|)+c A_{\lambda_{n}}^{\#} F(x) \leq c A_{c^{\prime} \lambda}^{\#} F(x),
\end{aligned}
$$

which proves (18).

Let $\widetilde{A}_{q} F=\left(\widetilde{A}\left(|F|^{q}\right)\right)^{1 / q}, q \geq 1$. Applying (6), (18) and the fact that $M_{\lambda}^{\#}\left(|f|^{1 / q}\right)(x) \leq\left(M_{\lambda}^{\#} f\right)^{1 / q}(x)$, we obtain

$$
\left(A_{q} F\right)^{*}(t) \leq\left(\widetilde{A}_{q} F\right)^{*}(t) \leq c \int_{t}^{\infty}\left(A_{q, \lambda}^{\#} F\right)^{*}(\tau) \frac{d \tau}{\tau} \quad\left(0<\lambda<\lambda_{n}\right)
$$

provided $(\widetilde{A} F)^{*}(+\infty)=0$. However, if $F$ is compactly supported, then $\widetilde{A}_{q} F$ is also compactly supported, and so $\left(\widetilde{A}_{q} F\right)^{*}(+\infty)=0$. Hence this latter assumption can be removed by taking an increasing sequence of functions $F_{k} \uparrow F$ with compact support, and using the fact that $\left|f_{k}\right| \uparrow|f|$ implies $f_{k}^{*}(t) \uparrow f^{*}(t)$ (see [2, p. 41]).

It remains to prove (3) for $\lambda_{n}<\lambda<1$. This follows immediately from (21) and the next lemma, which will also be crucial in the Proof of Theorem 2.2.

Define the $E$-functional by

$$
E_{q}(F, t)=\inf _{D}\left\|\mathcal{C}_{q}\left(F \chi_{D}\right)\right\|_{\infty}
$$

where the infimum is taken over all sets $D \subset \mathbb{R}_{+}^{n+1}$ with $\left|\operatorname{supp} A_{q}\left(F \chi_{D^{c}}\right)\right| \leq t$. 
Lemma 4.1. For any $0<\eta, \lambda<1$ and $0<q<\infty$ we have

$$
c_{1}\left(A_{q, \eta}^{\#} F\right)^{*}\left(c^{\prime} t / \eta\right) \leq E_{q}(F, t) \leq c_{2}\left(A_{q, \lambda}^{\#} F\right)^{*}\left(c^{\prime \prime} t\right) \quad(t>0),
$$

with constants $c^{\prime}, c^{\prime \prime}$ depending only on $n$, and constants $c_{1}, c_{2}$ depending on $n, \eta, \lambda$ and $q$.

Proof. Since $\left(A_{q, \eta}^{\#} F\right)^{*}(t) \leq\left(m_{\eta} A_{q} F\right)^{*}(t) \leq\left(A_{q} F\right)^{*}\left(t \eta / \gamma_{n}\right)$ and, by (2), $\left\|A_{q, \eta}^{\#} F\right\|_{\infty} \leq c\left\|\mathcal{C}_{q} F\right\|_{\infty}$, for any $D$ with $\left|\operatorname{supp} A_{q}\left(F \chi_{D^{c}}\right)\right| \leq t$ and $c^{\prime}=3 \gamma_{n}$ we have

$$
\begin{aligned}
\left(A_{q, \eta}^{\#} F\right)^{*}\left(c^{\prime} t / \eta\right) & \leq\left(\left(A_{\eta / 2}^{\#}\left(|F|^{q} \chi_{D^{c}}\right)+A_{\eta / 2}^{\#}\left(|F|^{q} \chi_{D}\right)\right)^{*}\left(c^{\prime} t / \eta\right)\right)^{1 / q} \\
& \leq\left(\left(A\left(|F|{ }^{q} \chi_{D^{c}}\right)\right)^{*}(3 t / 2)+c\left\|\mathcal{C}\left(|F|^{q} \chi_{D}\right)\right\|_{\infty}\right)^{1 / q} \\
& =c^{1 / q}\left\|\mathcal{C}_{q}\left(F \chi_{D}\right)\right\|_{\infty} .
\end{aligned}
$$

To prove the converse, define $\Omega=\left\{x: A_{q, \lambda}^{\#} F(x)>\left(A_{q, \lambda}^{\#} F\right)^{*}\left(c_{n} t\right)\right\}$, where $c_{n}$ is chosen so that $\left|\widetilde{\Omega}=\left\{x: M \chi_{\Omega}(x)>1 / 3^{n}\right\}\right| \leq t$. Now set $D=\cup_{x \in \widetilde{\Omega}^{c}} \Gamma(x)$. Then $\operatorname{supp} A_{q}\left(F \chi_{D^{c}}\right)=\widetilde{\Omega}$.

Let $B$ be an arbitrary ball. If $B \cap \Omega^{c} \neq \emptyset$, then, obviously,

$$
\left(\left(A_{q}\left(F \chi_{D} \mid r_{B}\right)\right) \chi_{B}\right)^{*}(\lambda|B|) \leq\left(A_{q, \lambda}^{\#} F\right)^{*}\left(c_{n} t\right) .
$$

If $B \subset \Omega$, then $3 B \subset \widetilde{\Omega}$, and thus $T(3 B) \subset D^{c}$. Since $\cup_{x \in B} \Gamma^{r_{B}}(x) \subset T(3 B)$, we get that $\left(\left(A_{q}\left(F \chi_{D} \mid r_{B}\right)\right) \chi_{B}\right)^{*}(\lambda|B|)=0$. Hence, by $(2)$,

$$
\left\|\mathcal{C}_{q}\left(F \chi_{D}\right)\right\|_{\infty} \leq c\left\|A_{q, \lambda}^{\#}\left(F \chi_{D}\right)\right\|_{\infty} \leq c\left(A_{q, \lambda}^{\#} F\right)^{*}\left(c_{n} t\right) .
$$

The lemma is proved.

Clearly, this lemma along with (21) implies (3) for any $0<\lambda<1$. Thus, the proof of Theorem 2.1 is complete.

Remark 4.2. Note that in [7] a Fefferman-Stein sharp function estimate of $\widetilde{A}(F)$ was obtained to give an alternate proof of (1).

Proof of Theorem 2.2. We have to prove only that

$$
K\left(F, t, T_{q}^{1}, T_{q}^{\infty}\right) \leq c \int_{0}^{t}\left(A_{q, \lambda}^{\#} F\right)^{*}(\tau) d \tau
$$

since the proof of the converse is easily follows from (5). Choose $D$ as in the proof of Lemma 4.1. Set $F_{1}=F \chi_{D^{c}}, F_{2}=F \chi_{D}$. Then we have $\left\|F_{2}\right\|_{T_{q}^{\infty}} \leq c\left(A_{q, \lambda}^{\#} F\right)^{*}\left(c_{n} t\right)$ and $\left|\operatorname{supp} A_{q}\left(F_{1}\right)\right| \leq t$. Thus $\left|\operatorname{supp} A_{q, \lambda}^{\#}\left(F_{1}\right)\right| \leq c t$, and hence (see $[\mathbf{2}$, p. 53]),

$$
\left\|A_{q, \lambda}^{\#}\left(F_{1}\right)\right\|_{1} \leq c^{\prime} \int_{0}^{c t}\left(A_{q, \lambda}^{\#} F\right)^{*}(\tau) d \tau
$$


From this and (5) we get

$$
\left\|F_{1}\right\|_{T_{q}^{1}}+t\left\|F_{2}\right\|_{T_{q}^{\infty}} \leq c^{\prime} \int_{0}^{c t}\left(A_{q, \lambda}^{\#} F\right)^{*}(\tau) d \tau,
$$

as required.

Proof of Proposition 2.3. It is easy to see that for any $h>0$,

$$
\left(\int_{\Gamma^{h}(\xi)}|F G(y, t)|^{q} \frac{d y d t}{t^{n+1}}\right)^{1 / q} \leq A_{\infty}(F)(\xi)\left(\int_{\Gamma^{h}(\xi)}|G(y, t)|^{q} \frac{d y d t}{t^{n+1}}\right)^{1 / q} .
$$

From this estimate and Proposition 3.1 we obtain

$$
\begin{aligned}
& \left(\left(A_{q}\left(F G \mid r_{B}\right)\right) \chi_{B}\right)^{*}(\lambda|B|) \\
& \leq\left(A_{\infty}(F) \chi_{B}\right)^{*}(\lambda|B| / 4)\left(\left(A_{q}\left(G \mid r_{B}\right)\right) \chi_{B}\right)^{*}(\lambda|B| / 4),
\end{aligned}
$$

which completes the proof.

Proof of Theorem 2.4. Since the function $M_{\lambda}^{\#} f$ is non-increasing in $\lambda$, it suffices to prove that

$$
M_{\lambda}^{\#} f(x) \leq c_{n, \lambda} S_{\lambda_{n} \lambda}^{\#} f(x) \quad(0<\lambda<1) .
$$

Then the statement of the theorem will follow for $0<\lambda<\lambda_{n}$. Assume first that $f \in \mathcal{S}$. Then (11) is equivalent to the fact that

$$
f(\xi)=\int_{0}^{\infty} f * \varphi_{t} * \psi_{t}(\xi) \frac{d t}{t} \quad \text { for all } \xi \in \mathbb{R}^{n} .
$$

Let $x, \xi \in Q$. Define $F(y, t)=f * \varphi_{t}(y)$ and set

$$
\begin{aligned}
f_{1, Q}(\xi) & =\int_{0}^{2 d_{Q}} \int_{\mathbb{R}^{n}} F(y, t) \psi_{t}(\xi-y) \frac{d y d t}{t} \\
f_{2, Q}(\xi) & =\int_{2 d_{Q}}^{\infty} \int_{\mathbb{R}^{n}} F(y, t)\left(\psi_{t}(\xi-y)-\psi_{t}\left(x_{Q}-y\right)\right) \frac{d y d t}{t} \\
C_{Q}(f) & =\int_{2 d_{Q}}^{\infty} \int_{\mathbb{R}^{n}} F(y, t) \psi_{t}\left(x_{Q}-y\right) \frac{d y d t}{t} .
\end{aligned}
$$

By (22), $f-C_{Q}(f)=f_{1, Q}+f_{2, Q}$. Using (19), (20) and Hölder's inequality, we get

$$
\left|f_{2, Q}(\xi)\right| \leq c \sum_{k=0}^{\infty} \frac{1}{2^{k}}\left(\left(A_{2}^{\prime}\left(F \mid 2^{k+2} d_{Q}\right)\right) \chi_{2^{k} Q}\right)^{*}\left(2^{k}|Q|\right) \equiv T_{1}(f ; Q) .
$$

We now estimate $\left(f_{1, Q} \chi_{Q}\right)^{*}(\lambda|Q|)$. Set $D=\bigcup_{x \in Q} \Gamma^{2 d_{Q}}(x)$ and

$$
\mathfrak{F}=\left\{x: A_{2}\left(F \chi_{D}\right)(x) \leq\left(A_{2}\left(F \chi_{D}\right)\right)^{*}\left(\lambda|Q| / 2 c_{n}\right)\right\},
$$

where $c_{n}$ is the same as in Lemma 3.2. Choose also $\mathfrak{F}^{*} \subset \mathfrak{F}$ as in Lemma 3.2. Let $E \subset Q$ be an arbitrary measurable set with $|E|=\lambda|Q|$. Then, arguing 
as in the proof of Lemma 3.3, we see that $\left|\mathfrak{F}^{*} \cap E\right| \geq \lambda|Q| / 2$. Next, let $D_{1}=\cup_{x \in \mathfrak{F}^{*} \cap E} \Gamma^{2 d_{Q}}(x)$ and

$$
\widetilde{f}(x)=\int_{0}^{2 d_{Q}} \int_{\mathbb{R}^{n}} F \chi_{D_{1}}(y, t) \psi_{t}(x-y) \frac{d y d t}{t} .
$$

Note that $f_{1, Q}(\xi)=\tilde{f}(\xi)$ for all $\xi \in \mathfrak{F}^{*} \cap E$. Write $\tilde{f}(\xi)=\sum_{Q_{Q^{\prime}}} a_{Q^{\prime}} \gamma_{Q^{\prime}}(\xi)$, where

$$
\begin{aligned}
\gamma_{Q^{\prime}}(\xi) & =\frac{1}{a_{Q^{\prime}}} \int_{\ell_{Q^{\prime}} / 2}^{\ell_{Q^{\prime}}} \int_{Q^{\prime}} F \chi_{D_{1}}(y, t) \psi_{t}(\xi-y) \frac{d y d t}{t} \\
a_{Q^{\prime}} & =c\left(\int_{\ell_{Q^{\prime} / 2}}^{\ell_{Q^{\prime}}} \int_{Q^{\prime}}\left|F \chi_{D_{1}}\right|^{2} d y \frac{d t}{t}\right)^{1 / 2}
\end{aligned}
$$

and the summation is carried over all dyadic cubes $Q^{\prime}\left(\ell_{Q^{\prime}}\right.$ denotes the side length of $Q^{\prime}$ ). Using the quasi-orthogonal argument (see [13, p. 171-172]), Lemma 3.2 , and the fact that $\operatorname{supp} A_{2}\left(F \chi_{D}\right) \subset 5 \sqrt{n} Q$, we get

$$
\begin{aligned}
\inf _{x \in E}\left|f_{1, Q}(x)\right| & \leq \inf _{x \in \mathfrak{F}^{*} \cap E}\left|f_{1, Q}(x)\right| \leq\left(\frac{c}{|Q|} \int|\widetilde{f}|^{2}\right)^{1 / 2} \\
& \leq c\left(\frac{1}{|Q|} \sum\left|a_{Q^{\prime}}\right|^{2}\right)^{1 / 2} \leq c\left(\frac{1}{|Q|} \int_{D_{1}}|F(y, t)|^{2} \frac{d y d t}{t}\right)^{1 / 2} \\
& \leq c\left(\frac{1}{|Q|} \int_{\mathfrak{F}} \int_{\Gamma(x)}\left|F \chi_{D}(y, t)\right|^{2} \frac{d y d t}{t^{n+1}} d x\right)^{1 / 2} \\
& \leq c\left(\left(A_{2}\left(F \mid 2 d_{Q}\right)\right) \chi_{5 \sqrt{n} Q}\right)^{*}\left(\lambda|Q| / 2 c_{n}\right) .
\end{aligned}
$$

Taking the supremum over all $E \subset Q$ with $|E|=\lambda|Q|$ gives

$$
\left(f_{1, Q} \chi_{Q}\right)^{*}(\lambda|Q|) \leq c\left(\left(A_{2}\left(F \mid 2 d_{Q}\right)\right) \chi_{5 \sqrt{n} Q}\right)^{*}\left(\lambda|Q| / 2 c_{n}\right) \equiv T_{2}(f ; Q) .
$$

Now let $f$ be an arbitrary function satisfying (10). Choose a sequence $f_{j} \in \mathcal{S}$ such that $\left(f_{j}\right)^{\#}(x) \leq c f^{\#}(x)$ and

$$
\int_{\mathbb{R}^{n}} \frac{\left|f(x)-f_{j}(x)\right|}{(1+|x|)^{n+1}} d x \rightarrow 0
$$

as $j \rightarrow \infty$. Using (13), (15) and (16), we obtain that

$$
T_{1}\left(f-f_{j} ; Q\right)+T_{2}\left(f-f_{j} ; Q\right) \rightarrow 0 \quad \text { as } \quad j \rightarrow \infty .
$$


Applying also (23), (24) and Lemma 3.3, we get

$$
\begin{aligned}
& \inf _{c}\left((f-c) \chi_{Q}\right)^{*}(\lambda|Q|) \\
& \leq \inf _{j}\left(\left(\left(f-f_{j}\right) \chi_{Q}\right)^{*}(\lambda|Q| / 2)+\left(\left(f_{j}-c_{Q}\left(f_{j}\right)\right) \chi_{Q}\right)^{*}(\lambda|Q| / 2)\right) \\
& \leq \inf _{j}\left\{\left(\left(f-f_{j}\right) \chi_{Q}\right)^{*}(\lambda|Q| / 2)+T_{1}\left(f-f_{j} ; Q\right)\right. \\
& \left.\quad+T_{2}\left(f-f_{j} ; Q\right)\right\}+c S_{\lambda_{n} \lambda}^{\#} f(x) \\
& =c S_{\lambda_{n} \lambda}^{\#} f(x) .
\end{aligned}
$$

The theorem is proved.

Proof of Corollary 2.5. Taking $\lambda=\lambda_{n}$ and applying then (14) along with (12) and (2), we obtain

$$
(f)_{2}^{\#}(x) \asymp M_{2}\left(M_{\lambda}^{\#} f\right)(x) \leq c M_{2}\left(S_{\lambda}^{\#} f\right)(x) \asymp \mathcal{T}^{\#} f(x) .
$$

To prove the converse, we need also the following simple estimate:

$$
M_{2}(M f(x)) \leq c M_{2} f(x) .
$$

In fact, this follows from elementary geometry of balls and from the $L^{2}$ boundedness of the Hardy-Littlewood maximal operator. We leave the details to the reader. Now, using (2), (13) and (25), we get

$$
\begin{aligned}
\mathcal{T}^{\#} f(x) \asymp M_{2}\left(S_{\lambda}^{\#} f\right)(x) & \leq c M_{2}\left(f^{\#}\right)(x) \asymp M_{2}\left(M M_{\lambda}^{\#} f\right)(x) \\
& \leq c M_{2}\left(M_{\lambda}^{\#} f\right)(x) \asymp(f)_{2}^{\#}(x),
\end{aligned}
$$

and this completes the proof.

Acknowledgements. The author thanks E. Liflyand for his interest in this work and helpful discussions. I am also grateful to the referee for his useful comments and suggestions.

\section{References}

[1] J. Alvarez and M. Milman, Interpolation of tent spaces and applications, SpringerVerlag Lecture Notes in Math., 1302 (1988), 11-21, MR 89i:46025, Zbl 0662.46076.

[2] C. Bennett and R. Sharpley, Interpolation of Operators, Academic Press, New York, 1988, MR 89e:46001, Zbl 0647.46057.

[3] W.S. Cohn and I.E. Verbitsky, Factorization of tent spaces and Hankel operators, J. Funct. Anal., 175 (2000), 308-329, MR 2001g:40247, Zbl 0968.46022.

[4] R.R. Coifman, Y. Meyer and E.M. Stein, Some new function spaces and their application to harmonic analysis, J. Funct. Anal., 62 (1985), 304-335, MR 86i:46029, Zbl 0569.42016. 
[5] C. Fefferman, Characterization of bounded mean oscillation, Bull. Amer. Math. Soc., 77 (1971), 587-588, MR 43 \#6713, Zbl 0229.46051.

[6] C. Fefferman and E.M. Stein, $H^{p}$ spaces of several variables, Acta Math., 129 (1972), 137-193, MR 56 \#6263, Zbl 0257.46078.

[7] E. Harboure, J.L. Torrea and B. Viviani, An application of the Fefferman-Stein inequality to the study of the tent spaces, Bull. London Math. Soc., 28 (1996), 161-164, MR 97a:42010, Zbl 0855.42010.

[8] B. Jawerth, The K-functional for $H^{1}$ and BMO, Proc. Amer. Math. Soc., 92 (1984), 67-71, MR 85j:42037, Zbl 0558.42013.

[9] B. Jawerth and A. Torchinsky, Local sharp maximal functions, J. Approx. Theory, 43 (1985), 231-270, MR 86k:42034, Zbl 0565.42009.

[10] F. John, Quasi-isometric mappings, Seminari 1962-1963 di Analisi, Algebra, Geometria e Topologia, Rome, 1965, MR 32 \#8315.

[11] A.K. Lerner, On weighted estimates of non-increasing rearrangements, East J. Approx., 4 (1998), 277-290, MR 99k:42043, Zbl 0947.42012.

[12] E.M. Stein, On the functions Littlewood-Paley, Lusin and Marcinkiewicz, Trans. Amer. Math. Soc., 88 (1958), 430-466, MR 22 \#3778, Zbl 0105.05104.

[13] Harmonic Analysis, Princeton Univ. Press, Princeton 1993, MR 95c:42002, Zbl 0821.42001.

[14] J.-O. Strömberg, Bounded mean oscillation with Orlicz norms and duality of Hardy spaces, Indiana Univ. Math. J., 28 (1979), 511-544, MR 81f:42021, Zbl 0429.46016.

Received December 24, 2001 and revised January 27, 2002.

Department of Mathematics

BAR-ILAN UNIVERSITY

52900 RAMAT GAN

ISRAEL

E-mail address: aklerner@netvision.net.il 